\title{
Adlayer structure and lattice size effects on catalytic rates predicted from KMC simulations: NO oxidation on $\mathrm{Pt}(111)$
}

Cite as: J. Chem. Phys. 149, 184701 (2018); https://doi.org/10.1063/1.5048787

Submitted: 17 July 2018 . Accepted: 16 October 2018 . Published Online: 12 November 2018

Konstantinos G. Papanikolaou, Matthew T. Darby (D), and Michail Stamatakis (D)

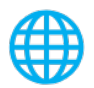

\section{ARTICLES YOU MAY BE INTERESTED IN}

\section{Field-theoretic simulations of bottlebrush copolymers}

The Journal of Chemical Physics 149, 184901 (2018); https://doi.org/10.1063/1.5051744

Perspective: Computational chemistry software and its advancement as illustrated through three grand challenge cases for molecular science

The Journal of Chemical Physics 149, 180901 (2018); https://doi.org/10.1063/1.5052551

Parallel kinetic Monte Carlo simulation framework incorporating accurate models of adsorbate lateral interactions

The Journal of Chemical Physics 139, 224706 (2013); https://doi.org/10.1063/1.4840395

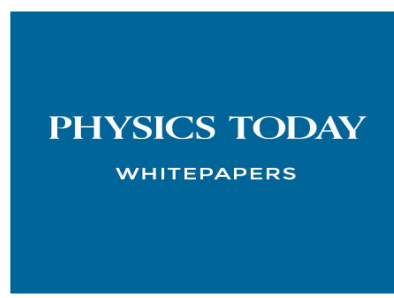




\title{
Adlayer structure and lattice size effects on catalytic rates predicted from KMC simulations: NO oxidation on $\mathrm{Pt}(111)$
}

\author{
Konstantinos G. Papanikolaou, Matthew T. Darby, and Michail Stamatakis ${ }^{\text {a) }}$ \\ Department of Chemical Engineering, University College London, Roberts Building, Torrington Place, \\ London WC1E 7JE, United Kingdom
}

(Received 17 July 2018; accepted 16 October 2018; published online 12 November 2018)

\begin{abstract}
Repulsive and/or attractive interactions between surface adsorbates have an important effect on the structure of the adsorbate layer and consequently on the rate of heterogeneous catalytic reactions. Thus, developing reaction models that take into account adsorbate-adsorbate interactions is crucial for making accurate predictions of the catalytic rate and surface coverage during reaction. In the present work, we employ kinetic Monte Carlo simulation to model the catalytic NO oxidation on Pt (111), adopting a cluster expansion (CE) Hamiltonian approach for treating the aforementioned interactions. We investigate CEs of increasing complexity, ranging from pairwise 1st nearest neighbor to long-range and many-body terms. We show that energetic models incorporating solely short-range interactions result in ordered adlayer structures, which are disrupted by anti-phase boundaries and defective regions when the size of the periodic lattice is non-commensurate to the structure of the stable adlayer. We find that $\mathrm{O}_{2}$ dissociates on sites located in these defective regions, which are predominantly responsible for the activity, and the predicted catalytic rate is strongly depended on the lattice size. Such effects are absent when employing non-periodic lattices, whereon the catalytic activity appears more intense on edges/corner sites. Finally, inclusion of long-range interactions in the model Hamiltonian induces relative disorder in the adsorbate layer, which is ascribed to the "softening" of the repulsive interactions between adspecies. Under these circumstances, the distribution of activation energies for $\mathrm{O}_{2}$ dissociation is broader as compared to short-range interaction models and on this basis we explain the disparate catalytic rate predictions when using different CEs. Published by AIP Publishing. https://doi.org/10.1063/1.5048787
\end{abstract}

\section{INTRODUCTION}

Catalytic processes are at the heart of the manufacturing of over $85 \%$ of everyday products currently on the market. ${ }^{1}$ The vast majority of these processes are heterogeneous, with a solid catalyst employed to catalyze the conversion of gaseous (or liquid) media, making these materials an indispensable part of the chemical industry. Indeed, the applications of solid heterogeneous catalysts are widespread, ranging from the production of fine and bulk chemicals to common plastics and hydrogen gas. ${ }^{2-4}$ Catalytic performance metrics, such as activity, selectivity, and yield, are related to the material chosen as the catalyst, thereby making the environmental and economic viability of the process dependent thereon.

Sophisticated statistical mechanics techniques, like kinetic Monte Carlo (KMC), ${ }^{5}$ aid our understanding with regard to important physico-chemical phenomena occurring during catalysis. Factors such as the structural intricacy of catalytic surfaces, the numerous elementary steps involved in the vast majority of chemical reactions, and lateral interactions between adsorbates raise the complexity of heterogeneous processes and affect the reaction kinetics. ${ }^{6,7}$ These crucial factors need to be taken into account to enable the accurate

a)Author to whom correspondence should be addressed: m.stamatakis@ ucl.ac.uk prediction of catalytic performance metrics, ${ }^{8}$ and this may be accomplished by employing on-lattice KMC models.

A complete kinetic theory should be able to predict the structure of the adsorbate layer over the catalytic surface and, according to that, accurately estimate the catalytic rate. ${ }^{9}$ Consequently, a large amount of effort has been dedicated toward gaining a fundamental understanding of thermodynamic and kinetic aspects in relation to adsorbate layers, including studies on phase transitions of single- and multispecies adlayers. ${ }^{10-13}$ Such studies often discuss the effect of adsorbate-adsorbate interactions on the adlayer structure, ${ }^{14-16}$ highlighting that these microscopic interactions affect the macroscopic catalytic properties of the system ${ }^{9}$ and are therefore of crucial importance in kinetic modeling. For example, adsorbate surface phenomena such as island formation in addition to well-defined, ordered overlayer structures have been verified through experimental and computational studies and are attributed to lateral interactions. ${ }^{14,17-20}$ By overlooking such interactions, it is often not possible to accurately reproduce the adsorbate layer structure, and their incorporation to kinetic models is often imperative to rationalize experimental observations and to accurately predict macroscopic observables. ${ }^{21,22}$

Macroscopic catalytic performance can be understood by considering the influence of lateral interactions on the microscale, especially, the resulting spatial correlations and 
the impact of lateral interactions on the activation energies of surface processes (adsorption, desorption, diffusion, and reaction). By the Brønsted-Evans-Polanyi (BEP) relation, ${ }^{23}$ a repulsive interaction between the initial state of a reaction and a spectator species will destabilize the initial state and thus reduce the activation barrier for the conversion to the final state species (we assume negligible interactions between the spectator and this final species, as would be the case for a gas product). In turn, such an interaction will raise the barrier for the reverse process (the opposite argument can be formed for an attractive lateral interaction). Since these effects depend on the nature and location of spectators around the reactants, an accurate model for the adsorbate overlayer energetics must be employed to reliably reproduce its structure. ${ }^{24-29}$

A versatile way to treat such interactions is through the use of the cluster expansion (CE) Hamiltonian method, ${ }^{30-32}$ as recently applied for catalytic rate predictions by Wu et al. ${ }^{22}$ Depending on the application, terms representing different adsorbate interactions can be included in the Hamiltonian, providing the opportunity to take into account interactions beyond the 1st nearest neighbor (1NN). This approach has been successfully used in numerous studies, ${ }^{21,33-35}$ having recently been incorporated in the graph theoretical KMC (GTKMC) framework of Stamatakis and Vlachos. ${ }^{36}$ Within this formalism, the total energy of the lattice is calculated from CE Hamiltonians by summing the energetic contributions of clusters that represent single-body to many-body adsorption configurations. The rates of elementary events are calculated with the aid of the aforementioned BEP relations, which relate the reaction energy (obtained from the cluster expansion) to the activation barrier of an event. ${ }^{37}$

In this work, we employ KMC simulation with CE energetics to investigate the effect of the oxygen adlayer structure on the estimated catalytic rate of a model reaction, namely, the catalytic oxidation of NO over $\operatorname{Pt}(111)$. The energetic contribution parameters of the various adsorbate clusters have been taken from the work of Schmidt et al. ${ }^{30}$ and are used in latticegas form. ${ }^{37}$ We study CEs of increasing complexity, ranging from $1 \mathrm{NN}$ to long range interactions, and we show that in the former case, the adlayer adopts an ordered $(\sqrt{3} \times \sqrt{3}) R 30^{\circ}$ structure at $1 / 3$ monolayer (ML) surface coverage for lattice sizes commensurate with this structure, thereby minimizing the repulsive oxygen-oxygen lateral interactions. Remarkably, for other lattice sizes, we observe the appearance of defective regions consisting of anti-phase boundaries and point defects, which form as a result of strong adatom-adatom interactions as well as the lattice periodicity, and considerably affect the predicted catalytic rate. Conversely, we find that the catalytic rate predictions become less dependent upon the lattice size when the catalytic oxidation is modeled on nonperiodic lattices (that are a better representation of individual nanoparticle facets) but also when the adsorbate layer becomes relatively disordered owing to the incorporation of long-range interactions.

The rest of the paper is organized as follows: in Sec. II, we present the background and the setup of the KMC calculations, providing details on the lateral interaction models and the reaction mechanism. We proceed by discussing the results obtained using different CE models on several lattices (periodic and non-periodic) in Sec. III. Finally, we summarize our findings and underline the significance of our work in Sec. IV.

\section{COMPUTATIONAL METHODS}

\section{A. Kinetic Monte Carlo background}

We have performed KMC simulations of NO oxidation on $\operatorname{Pt}(111)$ within the GT-KMC framework of Stamatakis and Vlachos, ${ }^{36}$ as implemented in Zacros (version 2.0). ${ }^{38}$ Adsorbate-adsorbate interactions are of high importance to our work and can be accurately captured by a general CE approach incorporated in this framework. The simulation input is composed of the reaction conditions (temperature, pressure, gas phase composition, etc.), the lattice structure that represents the catalytic surface, an energetic model where we specify the CE capturing the lateral interactions, and the reaction mechanism which defines all possible elementary events that can happen on the lattice. Optionally, one may also provide an initial adsorbate configuration on the lattice; otherwise, the simulation is by default initialized with an empty lattice.

The KMC algorithm starts by scanning the lattice and detecting all elementary processes that are possible given the current configuration. A lattice process queue is subsequently constructed, containing all the detected elementary processes along with their corresponding waiting times. The latter quantity is linked to the transition rate constant, which represents the average escape rate from system state $\sigma$ to another state $\sigma^{\prime}$, as expressed in the following equation: ${ }^{9,39}$

$$
\tau_{\text {trans }}^{\sigma \rightarrow \sigma^{\prime}}=-\frac{\ln (\chi)}{\mathrm{k}_{\sigma \rightarrow \sigma^{\prime}}},
$$

where $k_{\sigma \rightarrow \sigma^{\prime}}$ is the rate constant for transition from state $\sigma$ to state $\sigma^{\prime}$ and $\chi \in[0,1]$ is a uniformly distributed random number. After calculating the waiting times of the listed events, the one with the smallest value (i.e., the most imminent event) is identified. Subsequently, the system clock advances by $\min \left(\tau_{\text {trans }}^{\sigma \rightarrow \sigma^{\prime}}\right)$ and the most imminent event is executed, by modifying the adlayer structure and updating the lattice state and energetics accordingly. Elementary events that can no longer happen (as a result of the event just executed) are removed from the lattice process queue, and newly enabled processes are added to the list. The whole procedure of event selection, execution, and update is repeated, thereby generating a stochastic trajectory, which one can post-process to calculate quantities that can be experimentally evaluated (e.g., surface coverage and catalytic rates).

The frequency by which a particular event is executed during the simulation is proportional to its rate constant. The forward and reverse rate constants of each elementary event can be computed from the following equations, respectively:

$$
\begin{aligned}
& \mathrm{k}_{\mathrm{fwd}}=\frac{k_{B} \mathrm{~T}}{h} \frac{\mathrm{Q}^{\dagger}}{\mathrm{Q}_{\mathrm{R}}} \exp \left(-\frac{\mathrm{E}_{\mathrm{fwd}}^{\dagger}(\sigma)}{\mathrm{k}_{\mathrm{b}} \mathrm{T}}\right), \\
& \mathrm{k}_{\mathrm{rev}}=\frac{k_{B} \mathrm{~T}}{h} \frac{\mathrm{Q}^{\dagger}}{\mathrm{Q}_{\mathrm{P}}} \exp \left(-\frac{\mathrm{E}_{\mathrm{rev}}^{\dagger}(\sigma)}{\mathrm{k}_{\mathrm{b}} \mathrm{T}}\right),
\end{aligned}
$$


where $k_{B}$ and $h$ are Boltzmann's and Planck's constants, respectively; $k_{f w d}$ and $k_{\text {rev }}$ are the rate constants of the forward and reverse reactions, respectively; $\mathrm{Q}^{\dagger}, \mathrm{Q}_{\mathrm{P}}$, and $\mathrm{Q}_{\mathrm{R}}$ are the molecular partition functions of the transition state, products, and reactants, respectively; and $\mathrm{E}_{\mathrm{fwd}}^{\dagger}(\sigma)$ and $\mathrm{E}_{\mathrm{rev}}^{\dagger}(\sigma)$ are the activation energies of the forward and reverse reactions, respectively, which are affected by lateral interactions.

To account for the effect of lateral interactions on the reaction kinetics, the forward and reverse activation energies of each elementary reaction step are parameterized with respect to the reaction energy $\left(\Delta \mathrm{E}_{\mathrm{rxn}}\right)$ using a BEP relation. ${ }^{22,23}$ The latter quantity is estimated for each lattice state, $\sigma$, detected during the simulation and requires the calculation of the energy of configuration $\sigma$ and of the succeeding state $\sigma^{\prime}$ (for more information, see Sec. IV in the supplementary material of this work and Ref. 37). These configuration energies are in turn calculated using the CE Hamiltonian approach, ${ }^{31}$ as previously incorporated in the GT-KMC by Nielsen et al. ${ }^{37}$ Within this formalism, graph patterns representing single- to multi-body adsorbate configurations are treated as clusters, which contribute to the total energy of the lattice. To determine the total energy, the lattice is scanned by Zacros and clusters defined in the energetic model are detected and enumerated. ${ }^{37}$ The energy contribution of each cluster to a given lattice configuration is provided by a parameter known as the effective cluster interaction (ECI), and we compute the total energy of a lattice state $\sigma$ as

$$
H(\sigma)=\sum_{\mathrm{k}=1}^{N_{c}} \frac{\mathrm{ECI}_{\mathrm{k}}}{\mathrm{GM}_{\mathrm{k}}} \mathrm{NOC}_{\mathrm{k}},
$$

where $\mathrm{ECI}_{\mathrm{k}}$ is the effective cluster interaction of cluster $\mathrm{k}$, $\mathrm{GM}_{\mathrm{k}}$ is a graph multiplicity factor defined for $\mathrm{k}$ (included to prevent over-counting), and $\mathrm{NOC}_{\mathrm{k}}$ is the number of occurrences/instances of cluster k at state $\sigma$.

Finally, the catalytic rate is given by the turnover frequency (TOF) which is defined as the number of molecules of gas A produced per active site, per unit time. "A" is a representative gas species, which will be $\mathrm{NO}_{2}$ in our case (see Sec. II B). To determine the TOF, we plot the number of molecules of gas A produced during the simulation versus time and filter out any initial transients (whilst the system reaches steady state) before fitting using linear regression. Subsequently, we divide the slope by the total number of lattice sites (for further details and representative plots, see the supplementary material of this work) as shown in the following equation:

$$
\mathrm{TOF}=\frac{\mathrm{n}_{\mathrm{A}}}{\sum \tau \times \mathrm{N}_{\text {sites }}},
$$

where $n_{A}$ is the number of $\mathrm{A}$ molecules produced, $\sum \tau$ the simulation time, and $\mathrm{N}_{\text {sites }}$ the number of lattice sites.

\section{B. Reaction mechanism and simulation details}

Our work is based on an original reaction model devel-

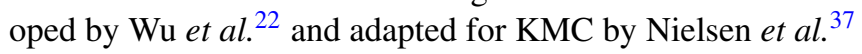
We explicitly consider three reversible elementary events [see Eqs. (6)-(8)], from which the $\mathrm{O}_{2}$ dissociation on $\mathrm{Pt}(111)$, Eq. (7), is the rate determining step (RDS). Furthermore, it is assumed that the catalytic oxidation of NO proceeds via an
Eley-Rideal mechanism and that oxygen adatoms $\left(\mathrm{O}^{*}\right)$ dominate the catalytic surface, in accord with the high efficiency of $\mathrm{NO}_{2}$ in supplying the catalyst surface with $\mathrm{O}$ atoms. ${ }^{40,41}$ Moreover, our analysis is restricted to surface coverage below $1 / 2 \mathrm{ML}$, where the Pt surface is not reconstructed, ${ }^{42}$ and $\mathrm{O}^{*}$ populate three-fold fcc hollow sites only $22,30,43$

$$
\begin{gathered}
\mathrm{NO}+\mathrm{O}^{*} \underset{k_{\text {red }}}{\stackrel{k_{\text {oxi }}}{\longrightarrow}} \mathrm{NO}_{2}+* \\
\mathrm{O}_{2}+2 * \underset{k_{\text {des }}}{\stackrel{k_{\text {ads }}}{\rightleftarrows}} \mathrm{O}^{*}+\mathrm{O}^{*}, \\
\mathrm{O}^{*}+* \underset{k_{\text {diff }}}{\stackrel{k_{\text {diff }}}{\rightleftarrows}} \mathrm{O}^{*}+*
\end{gathered}
$$

where * depicts a vacant fcc site. Equation (6) represents the reversible Eley-Rideal NO oxidation, Eq. (7) is the $\mathrm{O}_{2}$ dissociation on $\mathrm{Pt}(111)$, and Eq. (8) is the surface diffusion of $\mathrm{O}^{*}$ between neighboring fcc lattice sites.

As noted earlier, the forward and reversible rate constants of each elementary event are computed based on Eqs. (2) and (3), respectively, while the corresponding activation energies are found using a BEP relation (see the supplementary material). The proximity factor (defining the relative position of the transition state along the reaction coordinate) is set to unity for the dissociation of $\mathrm{O}_{2}$ [Eq. (7)] as the transition state is molecular $\mathrm{O}_{2}$ with an elongated $\mathrm{O}-\mathrm{O}$ bond, ${ }^{22}$ zero for the $\mathrm{NO}$ oxidation/reduction [Eq. (6)], and 0.5 for $\mathrm{O}^{*}$ diffusion [Eq. (8)]. Furthermore, the activation energies at the zero coverage limit for the different CEs are found in Sec. III of the supplementary material.

In relation to the rate constants, there is a quasiequilibrium where the dynamics of $\mathrm{NO}$ oxidation/reduction and $\mathrm{O}^{*}$ diffusion are fast compared to $\mathrm{O}_{2}$ dissociation, such that the latter is the RDS (based on the previous work by Schneider and co-workers). ${ }^{22}$ We therefore provide values of the pre-exponential terms for the NO oxidation/reduction and $\mathrm{O}^{*}$ diffusion such that the rates of these elementary events are at least 50 times larger than the rate of the RDS, assuring the fast equilibration of the adlayer. ${ }^{37,44}$ This method has been implemented in our previous work, and for further details on the derivation and calculation of the rate constants, the reader may consult Ref. 37.

Within GT-KMC, the catalytic surface is represented as a two-dimensional lattice graph where vertices denote surface sites, and edges define the connectivity (neighboring relations) between those sites. We perform simulations using two types of lattices: with and without periodic boundary conditions. The shapes of the periodic lattices are equilateral parallelograms of dimensions $\mathrm{m} \times \mathrm{m}$ (containing a total of $\mathrm{m}^{2}$ sites), and for the non-periodic ones, we use equilateral parallelograms in addition to equilateral triangles of side length $\mathrm{n}$ (containing a total of $\sum_{\mathrm{i}=1}^{\mathrm{n}} \mathrm{i}$ sites).

The reaction is modeled at a total pressure of 1 bar and fixed partial pressures of $\mathrm{NO}, \mathrm{O}_{2}$, and $\mathrm{NO}_{2}$. The $\mathrm{NO}_{2}$ to $\mathrm{NO}$ pressure ratio is set to $0.37 \mathrm{in}$ all simulations, thereby fixing the chemical potential of surface oxygen $\mu_{\mathrm{O}^{*}}$ [since reaction (6) is fast], and the partial pressure of $\mathrm{O}_{2}$ is set to 0.1 . We run two sets of simulations for low $(480 \mathrm{~K})$ and high $(680 \mathrm{~K})$ temperatures, and the lattice is always initialized with an $\mathrm{O}^{*}$ adsorbate 


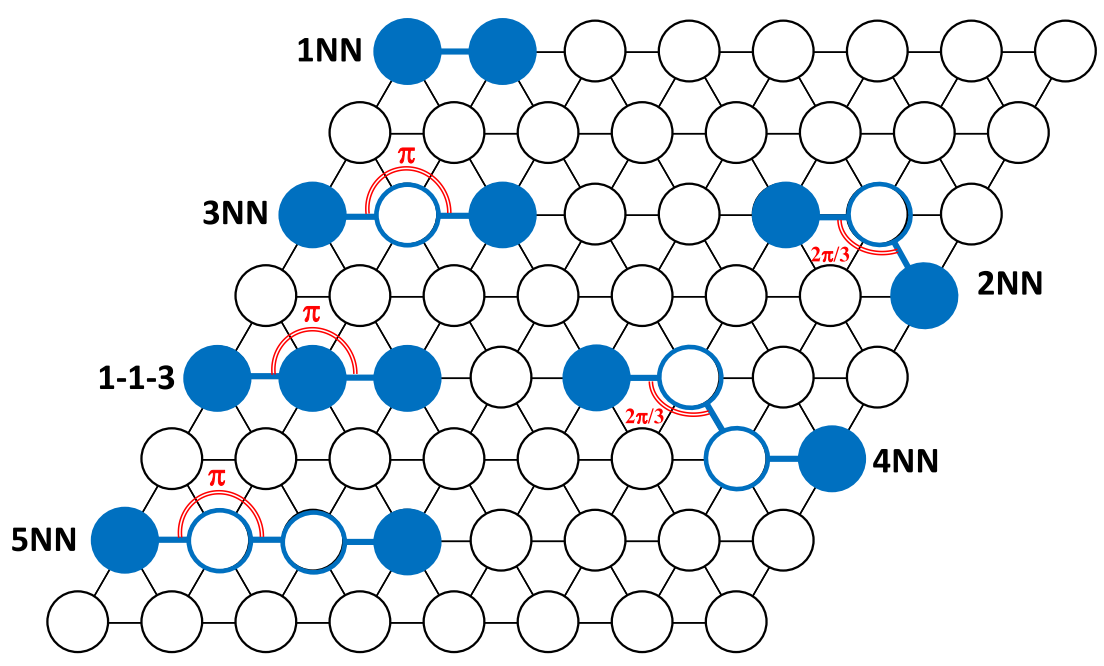

FIG. 1. Graph patterns (or clusters) that are taken into account in 3-Fig., 5-Fig., and 8-Fig. CE Hamiltonian models. The blue circles indicate sites occupied by oxygen atoms. The white circles with black edges are vacant sites, and white circles with blue edges indicate sites that may or may not be vacant.

overlayer with the $(\sqrt{3} \times \sqrt{3}) R 30^{\circ}$ structure, which is a stable adlayer configuration when using short-range, $1 \mathrm{NN}$ lateral energetics at $1 / 3 \mathrm{ML}$ coverage. Thus, the system is initially in a low energy state, although it is allowed to reaching stationary conditions before any analysis of the kinetics is carried out.

\section{Lateral interaction models}

We study different CE models of varying complexity. In particular, we examine cases where the $\mathrm{CE}$ Hamiltonian contains 3, 5, and 8 figures (clusters) and the corresponding graph patterns are presented in Fig. 1. Each Hamiltonian contains a constant term (which does not affect any of the results but is included for compatibility with other Hamiltonian descriptions) and a single-body term that models the adsorption energy of one $\mathrm{O}^{*}$ adsorbate. In the 3-Fig. CE model, two-body interactions with adsorbate separation of up to $1 \mathrm{NN}$ are considered; these short range interactions are often referred to as hard interactions herein, as they lead to highly ordered adlayer structures (see Sec. III). In addition to $1 \mathrm{NN}$ interactions, $2 \mathrm{NN}$ and $3 \mathrm{NN}$ two-body interactions are included in the 5-Fig. CE model (Fig. 1). Finally, the 8-Fig. CE model incorporates the clusters found in the 3-Fig. and 5-Fig. cluster expansions, as well as two-body terms at $4 \mathrm{NN}$ and $5 \mathrm{NN}$ separation in addition to a 3-body NN cluster (1-1-3 cluster in Fig. 1). The terms included in each CE model along with their ECIs can be found in Table S1 in the supplementary material.

\section{RESULTS AND DISCUSSION}

\section{A. 3-Figure CE energetics}

We first examine a 3-Fig. CE model where only 1NN interactions contribute to the energetics. To exclude latticeboundary effects on the predicted catalytic rate, we have performed simulations on periodic equilateral lattices of various sizes at the two aforementioned temperatures $(480 \mathrm{~K}$ and $680 \mathrm{~K})$. The calculated TOFs on parallelogram lattices with the number of sites ranging between 36 and $2500(6 \times 6$ to $50 \times 50)$ are plotted in Fig. 2. For both temperatures, there is an oscillatory behavior in the catalytic rate as the lattice size is increased. We observe a regular pattern by which certain lattice sizes exhibit much higher activities than others, a difference which does not diminish even for relatively large lattices of more than 2000 sites. Upon further inspection, we note that lattices with a number of sites per side that is divisible by 3 exhibit a significantly lower TOF (ca. one order of magnitude lower) than those with sites non-divisible by this number.

As highlighted earlier in our discussion on the BEP relationship, the activation energy for a surface reaction is dependent on the configuration of neighboring spectators in the adsorbate overlayer. Consequently, both the kinetic constant and the catalytic rate also depend on the configuration of spectators. Thus, we can elucidate the variability in the values of TOF by studying the adlayer structures of different lattices giving high and low TOF. To this end, we use two representative lattice sizes, a $42 \times 42$ lattice (1764 sites), whose number of sites per side is divisible by 3 , and a $44 \times 44$ lattice $(1936$ sites), for which this is not true. The snapshots of these lattices for simulations at $480 \mathrm{~K}$ are shown in Figs. 3(a) and 3(b), respectively.

In a representative configuration at stationary conditions on the $42 \times 42$ lattice, the $\mathrm{O}^{*}$ adspecies are perfectly ordered in the $(\sqrt{3} \times \sqrt{3}) R 30^{\circ}$ structure, which minimizes the $1 \mathrm{NN}$

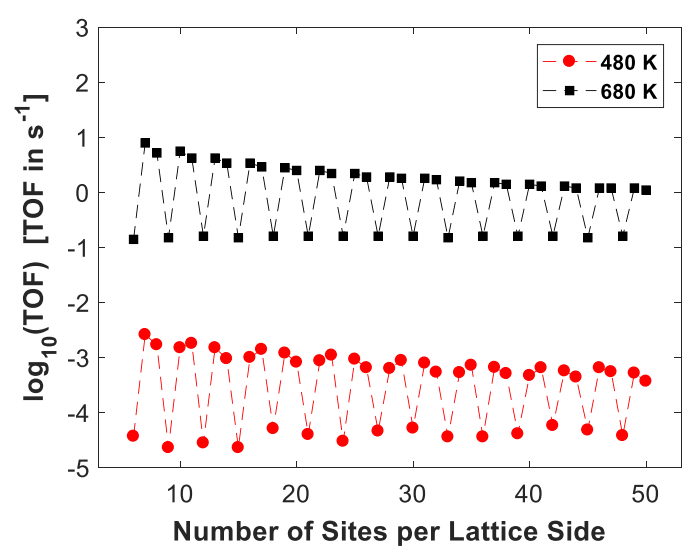

FIG. 2. Computed TOFs of the 3-Fig. CE model for a range of lattice sizes at $480 \mathrm{~K}$ (red) and $680 \mathrm{~K}$ (black). $\mathrm{P}_{\text {tot }}=1$ bar, $\mathrm{P}_{\mathrm{NO}_{2}} / \mathrm{P}_{\mathrm{NO}}=0.37$, and $\mathrm{y}_{\mathrm{O}_{2}}=0.1$ for all simulations. 

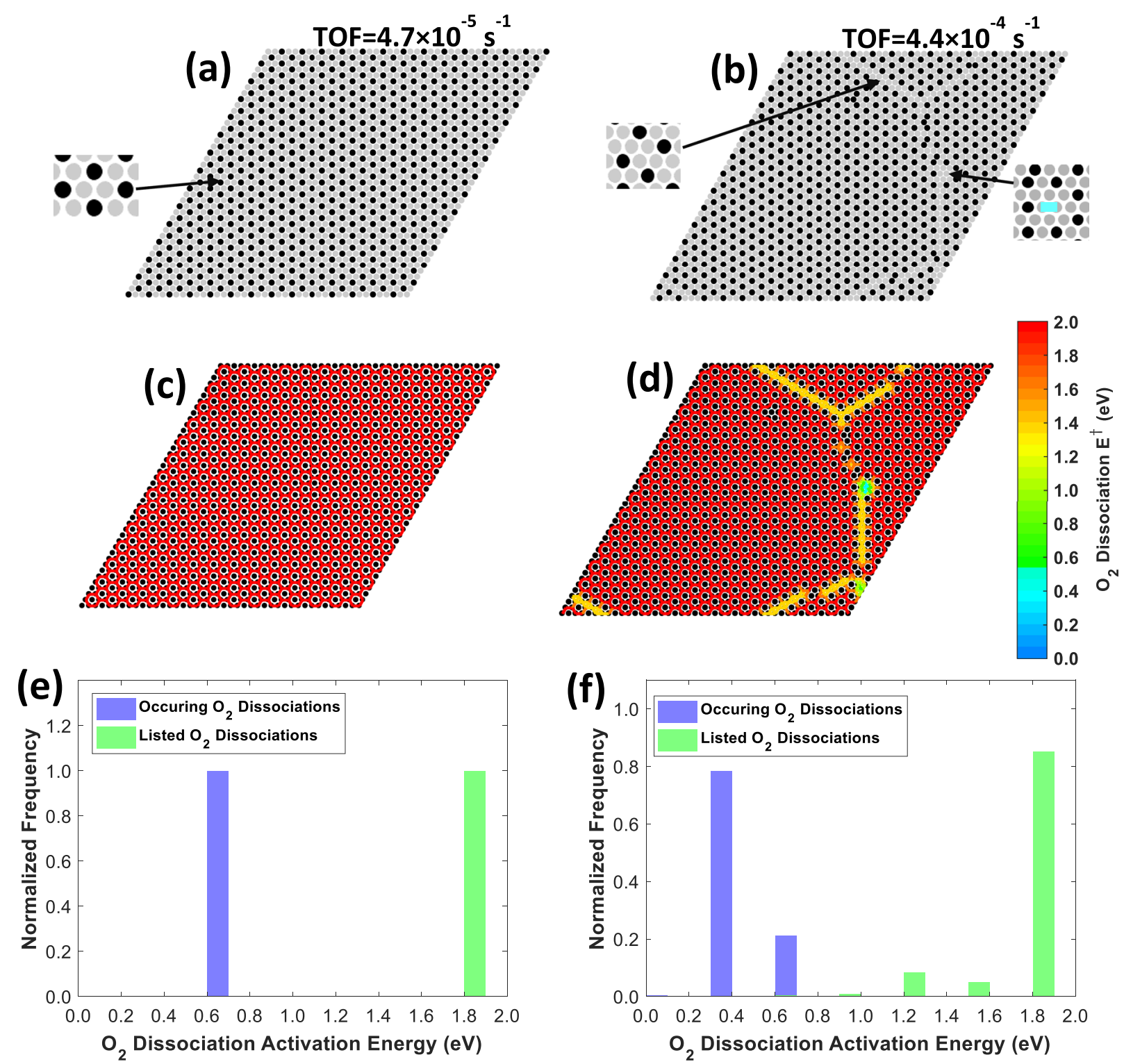

FIG. 3. Representative lattice snapshots from KMC simulation at $480 \mathrm{~K}$ of a 3-Fig. CE energetic model. Panels (a) and (b) show the adlayer structure of a 42 $\times 42$ and a $44 \times 44$ lattice, respectively; $\mathrm{O}^{*}$ adatoms are represented by black circles and empty sites are represented by gray circles. The close-up on the left of panel (a) shows a phase domain local configuration, while close-ups on the left and right of panel (b) indicate the local anti-phase boundary and point defect configurations, respectively. Panels (c) and (d) show the same snapshots but colored according to the local $\mathrm{O}_{2}$ dissociation activation energy where low values and high values are shown in blue and red, respectively. In panel (e), the green histogram shows the distribution of activation energies of $\mathrm{O}_{2}$ dissociation for all events listed by the KMC algorithm in the configuration (KMC snapshot) of panel (a) $(42 \times 42$ lattice). On the other hand, the blue histogram of panel (e) shows the distribution of activation energies of the actually executed adsorptions throughout the simulation. Panel (f): as panel (e) but for the KMC snapshots of panel (b) for the $44 \times 44$ lattice. The former are normalized to the total number of listed $\mathrm{O}_{2}$ dissociations for the particular KMC snapshot, and the latter to the total number of executed $\mathrm{O}_{2}$ dissociations throughout the $\mathrm{KMC}$ simulation. $\mathrm{P}_{\mathrm{tot}}=1 \mathrm{bar}, \mathrm{P}_{\mathrm{NO}_{2}} / \mathrm{P}_{\mathrm{NO}}=0.37$, and $\mathrm{y}_{\mathrm{O}_{2}}=0.1$ bar.

repulsions exerted upon them [Fig. 3(a)]. The ordered structure is, however, disrupted on the $44 \times 44$ lattice by the formation of anti-phase boundaries that appear to divide the adlayer into a number of perfect $(\sqrt{3} \times \sqrt{3}) R 30^{\circ}$ domains [Fig. 3(b)]. As the $44 \times 44$ lattice has a number of sites per side that is not divisible by 3 , it is impossible for the adlayer to organize into a perfect $(\sqrt{3} \times \sqrt{3}) R 30^{\circ}$ structure and instead forms line-defects: the observed anti-phase boundaries. In other words, the size of the lattice is not commensurate to the stable $(\sqrt{3} \times \sqrt{3}) R 30^{\circ}$ adlayer structure. Closer examination of these defects reveals lower local coverage in the anti-phase boundary [Fig. 3(b), inset left] compared to the ordered $(\sqrt{3} \times \sqrt{3}) R 30^{\circ}$ domain [Fig. 3(a), inset]. Even lower local $\mathrm{O}^{*}$ coverages are detected on pointdefect regions that are adjacent to anti-phase boundaries [Fig. 3(b), inset right], where remarkably, the pair of fcc sites covered by the cyan line is surrounded by just one neighboring $\mathrm{O}^{*}$ adatom.

The local coverage affects the activation barrier of the RDS (i.e., $\mathrm{O}_{2}$ dissociation) as a result of the repulsion between $\mathrm{O}^{*}$ adatoms. By the BEP relationship, the lower coverage of $\mathrm{O}^{*}$ in the anti-phase boundary is expected to result in lower activation barriers on fcc site pairs thereon. The effect of the local coverage on the $\mathrm{O}_{2}$ dissociation activation is shown in 
Figs. 3(c) and 3(d), where the snapshots found in Figs. 3(a) and 3(b) are color-coded, respectively, according to the activation energy values of the dissociation of $\mathrm{O}_{2}$. The presence of four $1 \mathrm{NN} \mathrm{O}^{*}$ adatoms around empty fcc site pairs in the $(\sqrt{3} \times \sqrt{3}) R 30^{\circ}$ phase domain considerably hampers the dissociation of $\mathrm{O}_{2}$, by significantly elevating the activation barriers from $0.02 \mathrm{eV}$ at the zero coverage limit (see the supplementary material) $)^{37}$ to approximately $1.9 \mathrm{eV}$ in this region. On the other hand, the lower local coverage in anti-phase boundary and point defect configurations results in lower values for the activation energy, between 1.2 and $1.6 \mathrm{eV}$ at the former and less than $1 \mathrm{eV}$ at the latter. Consequently, in these regions, high values of the $\mathrm{O}_{2}$ dissociation rate constant are computed, making the RDS more probable to occur.

However, the different adlayer regions seen in Fig. 3(d) do not equally contribute to the catalytic rate. To identify the local configurations whereby oxygen adsorptions occur on each lattice, we plot a histogram of the distribution of $\mathrm{O}_{2}$ dissociation activation energies on the $42 \times 42$ and $44 \times 44$ lattices in Figs. 3(e) and 3(f), respectively. The green bars in these histograms show the activation energy distribution of $\mathrm{O}_{2}$ dissociation events that may occur on each lattice [Figs. 3(a) and 3(b)]. These are possible events that may happen on vacant fcc site pairs and are listed in the event-queue of the KMC algorithm. As expected, in the $42 \times 42$ system, all of these listed events have the same activation energy $(\sim 1.9 \mathrm{eV})$ because of the ordered $(\sqrt{3} \times \sqrt{3}) R 30^{\circ}$ structure seen in Fig. 3(a). This is not true for the $42 \times 42$ lattice as a result of the observed antiphase boundaries. In this case, the majority of $\mathrm{O}_{2}$ dissociations belong to the ordered $(\sqrt{3} \times \sqrt{3}) R 30^{\circ}$ phase simply because this phase covers most of the lattice area and has activation energies of 1.8-1.9 eV [the tallest bar on the right of Fig. 3(f)]. Events with activation energies between 1.2 and $1.6 \mathrm{eV}$ correspond to adsorption processes on anti-phase boundary sites, while those below $1.0 \mathrm{eV}$ correspond to $\mathrm{O}_{2}$ dissociations on sites located in point defect regions. The normalized frequencies of possible dissociation events on anti-phase boundary and point defects are less than $19 \%$ and $4 \%$, respectively, of that over the ordered domain.
On the other hand, the blue histograms shown in Figs. 3(e) and 3(f) correspond to $\mathrm{O}_{2}$ dissociation events that actually occur (i.e., they are executed by the KMC algorithm during the simulation) on the $42 \times 42$ and $44 \times 44$ lattices, respectively. To generate these histograms, we record the activation energies of all occurring $\mathrm{O}_{2}$ dissociations throughout the simulation. Interestingly, on the $44 \times 44$ lattice, no $\mathrm{O}_{2}$ dissociation happens on $(\sqrt{3} \times \sqrt{3}) R 30^{\circ}$ and anti-phase boundary domains [Fig. 3(f)]; instead, these events happen primarily at point defects adjacent to the anti-phase boundaries, with the largest portion of the lattice remaining inactive. The catalytic activity is dominated by defect-regions that contain fcc site pairs with two $1 \mathrm{NNs}$ or less, and the overall reaction appears to proceed in short "bursts" in these highly active but also highly ephemeral point defects. Thus, the absence of anti-phase boundaries in the $42 \times 42$ lattice, where all $\mathrm{O}_{2}$ dissociations happen on rarely formed point defects with an activation barrier of $0.62 \mathrm{eV}$ [Fig. 3(e)], explains the one order of magnitude lower TOF compared to that on the $44 \times 44$ lattice, as well as the fluctuations in the catalytic rate as shown in Fig. 2.

\section{B. NO oxidation on non-periodic lattices}

To verify whether the discussed effects in Sec. III A are associated with the lattice periodicity, we have performed additional simulations using non-periodic equilateral parallelogram lattices. The latter provide a better description of complete nanoparticle facets. By eliminating the periodic boundary condition, we break the connectivity between sites that belong to the opposite sides of the lattice. Consequently, potential reaction patterns that involve $\mathrm{O}^{*}$ (or vacant sites) located on two opposite sides cannot be detected by the algorithm in this case. We observe that under these circumstances, there is no generation of anti-phase boundaries that disrupt the $(\sqrt{3} \times \sqrt{3}) \mathrm{R} 30^{\circ}$ structure, and therefore, the catalytic rate is not subject to systematic changes at increasing lattice size as in Fig. 2 [Fig. 4(a)].

On the contrary, we find that the computed TOF decreases with increasing number of lattice sites for lattices smaller than $46 \times 46$ [Fig. 4(a)]. Beyond that point, the catalytic rate is prac-
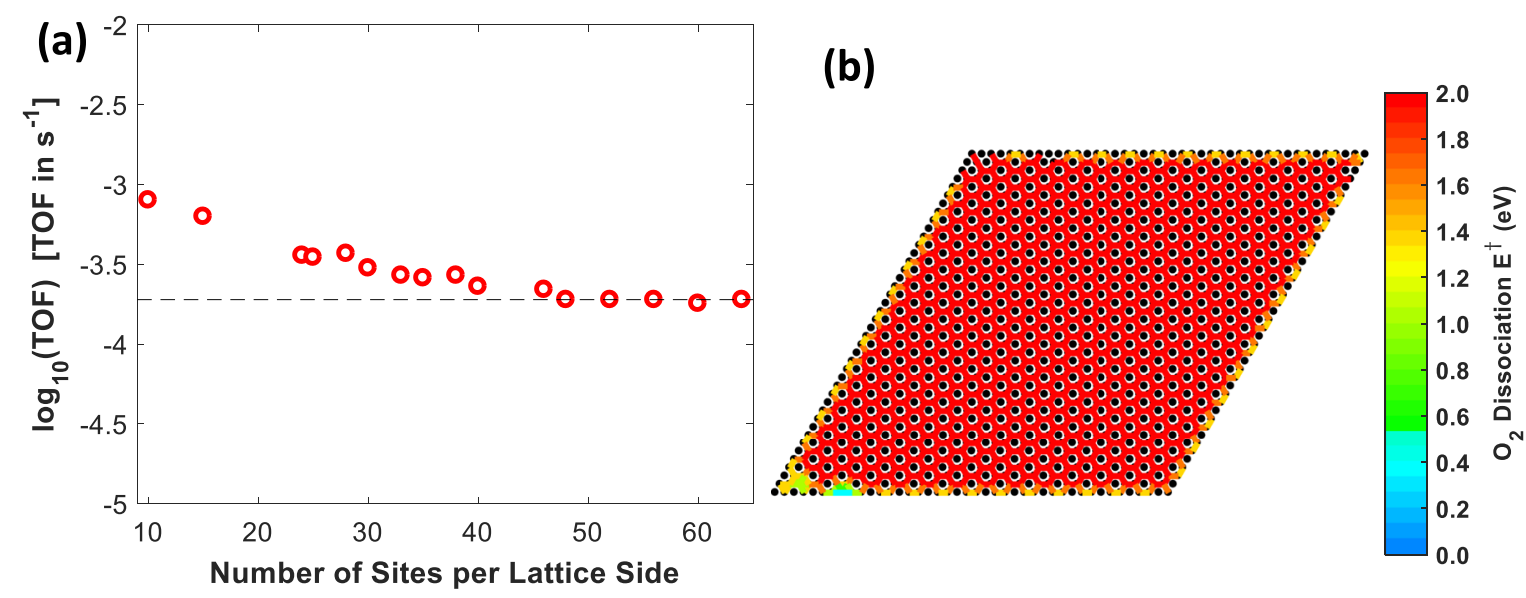

FIG. 4. (a) TOF of various non-periodic parallelogram lattices at $480 \mathrm{~K}, \mathrm{y}_{\mathrm{O}_{2}}=0.1$ bar, and $\mathrm{P}_{\mathrm{NO}} / \mathrm{P}_{\mathrm{NO}}=0.37$ for 3 -Fig. CE energetics. (b) $\mathrm{O}_{2}$ dissociation color-coded KMC snapshot of a $42 \times 42$ lattice, where low values and high values are shown in blue and red, respectively. Conditions are as in panel (a). 
tically constant and independent of the lattice size [Fig. 4(a)]. Notwithstanding, highly active point defects are now present on the lattice edges/corners throughout the KMC simulation [Fig. 4(b)]. As a result of the non-periodicity, adspecies on edges/corners experience less repulsion compared to the inner phase adsorbates, as it may be true in a real nanoparticle facet. More specifically, the "curvature" at nanoparticle edges results in larger distances between adsorbates in neighboring facets as compared to cases where these adsorbates are on a planar surface. In addition, edge adsorbates might be differently oriented from the adsorbates on conterminous facets, ${ }^{45}$ and therefore, the dynamics of non-periodic lattices may better represent those on the facets of a nanoparticle.

In line with the latter consideration, we model the catalytic oxidation on equilateral triangular (111) lattices that have the same shapes as facets encountered in common nanoparticle structures such as cube-octahedral and octahedral. Figure 5 shows the results of such $\mathrm{KMC}$ simulations, revealing that $\mathrm{O}_{2}$ dissociation is indeed more facile on edges/corners as compared to the inner parts of the lattice, and therefore, the catalytic activity emerges predominantly from such locations in our non-periodic 3-Fig. CE calculations.

The edge/corner sites are also characterized by a distinct electronic environment as a result of their low coordination number (see, e.g., Ref. 46), which is not taken into account in our simulations. What the latter show is that, even in the absence of such electronic effects, there is a remarkably different physicochemical environment on edges/corners that is governed by geometry and lateral interaction strength, which results in the distinct behavior of these sites compared to those in the inner part of the facet. It should, however, be noted that for a complete description of the chemistry toward highly accurate catalytic rate predictions on edge and corner sites, electronic effects have to be accounted for, in addition to the geometric effects shown here. The adsorption energy of $\mathrm{O}^{*}$ at edges and terraces is often not the same. ${ }^{46}$ This is verified by the higher TOF measured at larger Pt particle size, which implies that edge/corner sites might be poisoned in practice as a result of strong adsorbate binding. ${ }^{47}$

\section{Beyond 1NN interactions}

We proceed by investigating the effect of long-range interactions on the adlayer structure. We will first present the results for a 5-Fig. CE Hamiltonian model that includes long range adsorbate-adsorbate interactions ( $2 \mathrm{NN}$ and $3 \mathrm{NN}$ ) and leads to more accurate predictions than the 3-Fig. CE. ${ }^{37}$ We instantly note significant differences between the results of these simulations (Fig. 6) compared to those previously obtained with the 3-Fig. CE (Fig. 3). We show simulation snapshots with color-coded maps of the $\mathrm{O}_{2}$ dissociation barriers, on a $44 \times 44$ non-commensurate periodic lattice at $480 \mathrm{~K}$ and $680 \mathrm{~K}$ [Figs. 6(a) and 6(b), respectively]. There is a notable absence of well-defined anti-phase boundary regions in these cases, as a result of incorporating $2 \mathrm{NN}$ and $3 \mathrm{NN}$ lateral interactions (Fig. 6), and the adlayer distribution appears more random compared to that obtained by the 3-Fig. CE at $480 \mathrm{~K}$ [Fig. 6(a)]. In turn, the calculated TOF is significantly higher due to the presence of several highly active configurations characterized by low local coverage, with $\mathrm{O}_{2}$ dissociation activation barriers less than $1.0 \mathrm{eV}$ [Fig. 6(c)].

At $480 \mathrm{~K}$, we observe some $(\sqrt{3} \times \sqrt{3}) \mathrm{R} 30^{\circ}$ islands in the $\mathrm{O}^{*}$ adlayer, and the total $\mathrm{O}^{*}$ surface coverage is around 0.34 ML. As seen from the color-coded KMC snapshot in Fig. 6(a), the $\mathrm{O}_{2}$ dissociation activation barrier on fcc pairs belonging to these ordered islands is approximately $1.4 \mathrm{eV}$. This lower activation energy compared to that of the 3-Fig. $\mathrm{CE}$ on identical adsorbate configurations (i.e., $1.9 \mathrm{eV}$; see Fig. 3) reflects the "softening" of adsorbate-adsorbate interactions at higher order CEs. The distribution of activation energies for the executed $\mathrm{O}_{2}$ dissociations at $480 \mathrm{~K}$ [Fig. 6(c) blue bins] reveals that no oxygen adsorption occurs on $(\sqrt{3} \times \sqrt{3}) \mathrm{R} 30^{\circ}$ islands. On the contrary, around $40 \%$ of $\mathrm{O}_{2}$ dissociation events happen with activation energies between 0.4 and $0.5 \mathrm{eV}$, in configurations with no more than two $1 \mathrm{NN}$ $\mathrm{O}^{*}$ adatoms [for example, the configuration highlighted by the cyan line in the inset above the blue bin at $0.4-0.42 \mathrm{eV}$ in Fig. 6(c)].

Furthermore, at $680 \mathrm{~K}$, the $\mathrm{O}^{*}$ adlayer appears mostly random, as it lacks any appreciably large ordered island [Fig. 6(b)]. A lower average $\mathrm{O}^{*}$ coverage $(\sim 0.27 \mathrm{ML})$ and a greater degree of adlayer disorder compared to the previous case at $\mathrm{T}=480 \mathrm{~K}$ give rise to numerous local adlayer configurations of very low coverage, which encompass pairs of fcc sites without any $1 \mathrm{NN} \mathrm{O}^{*}$ adatoms. Unsurprisingly, the majority of $\mathrm{O}_{2}$ dissociations happen therein and are executed with activation energies below $0.1 \mathrm{eV}$ [Fig. 6(c)—green bins]. The occurrence of these low local coverage configurations in conjunction with the relatively high temperature results in higher TOF by two orders of magnitude as compared to $480 \mathrm{~K}$.

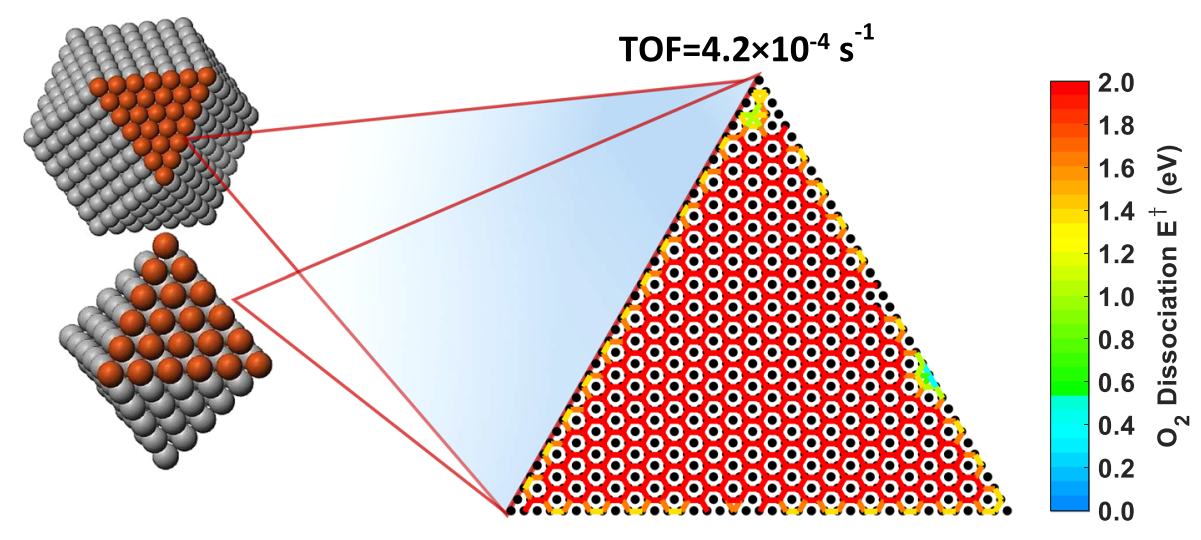

FIG. 5. Typical snapshot of a KMC simulation using a triangular nonperiodic lattice with 780 sites of a 3-Fig. $\mathrm{CE}$ energetic model at $480 \mathrm{~K}$ and colorcoded according to $\mathrm{O}_{2}$ dissociation activation energy (low and high values are shown in blue and red, respectively). Nanoparticle facets of similar geometry are found in octahedral and cubeoctahedral particles shown on the left of the figure. $\mathrm{P}_{\text {tot }}=1$ bar, $\mathrm{P}_{\mathrm{NO}_{2}} / \mathrm{P}_{\mathrm{NO}}$ $=0.37$, and $\mathrm{y}_{\mathrm{O}_{2}}=0.1$ bar. 

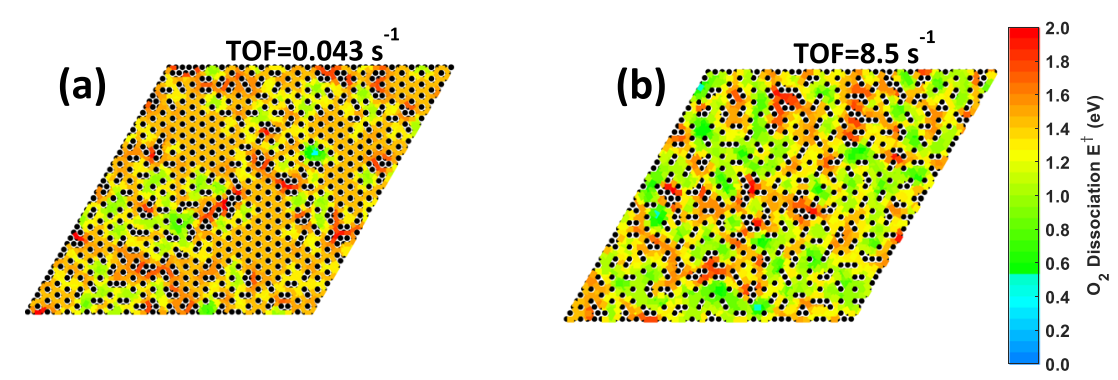

(c)

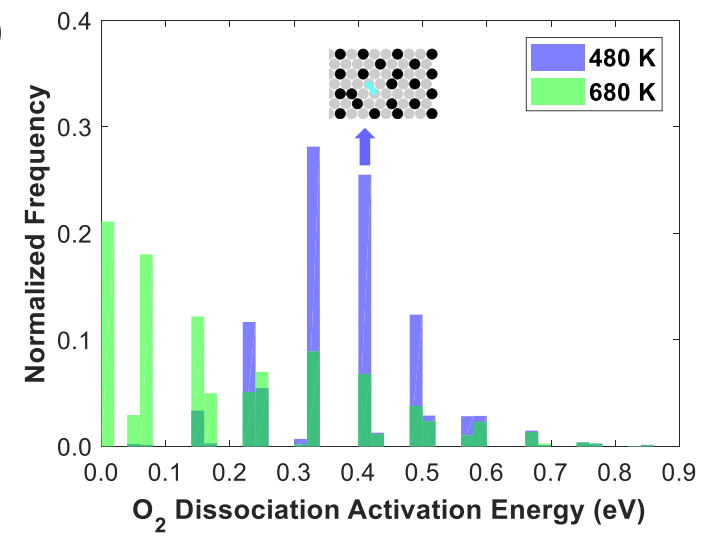

Instead of a "short burst" behavior, as for the 3-Fig. CE (Fig. 3), events are executed with a broader range of activation energies at both temperatures with a "widespread participation" of fcc site pairs in the catalytic rate [Fig. 6(c)].

Plots of the computed TOFs at $480 \mathrm{~K}$ and $680 \mathrm{~K}$ for lattice sizes up to 2500 sites are given in Fig. 7. We note a key difference between the simulations at $680 \mathrm{~K}$ and $480 \mathrm{~K}$; at $680 \mathrm{~K}$, there is near-constant TOF with increasing lattice size, whereas at $480 \mathrm{~K}$ there are systematic variations of the catalytic rate. These fluctuations at $480 \mathrm{~K}$ span a narrower range as compared to those of 3-Fig. CE (see Fig. 2) and are associated with the retained spatial correlation at this temperature. Upon closer inspection, we also note that the predicted TOF on commensurate lattices at $480 \mathrm{~K}$ exhibits an increasing trend with increasing lattice size (Fig. 7), although this trend fades for larger lattices, beyond $45 \times 45$ (Fig. 7). This trend

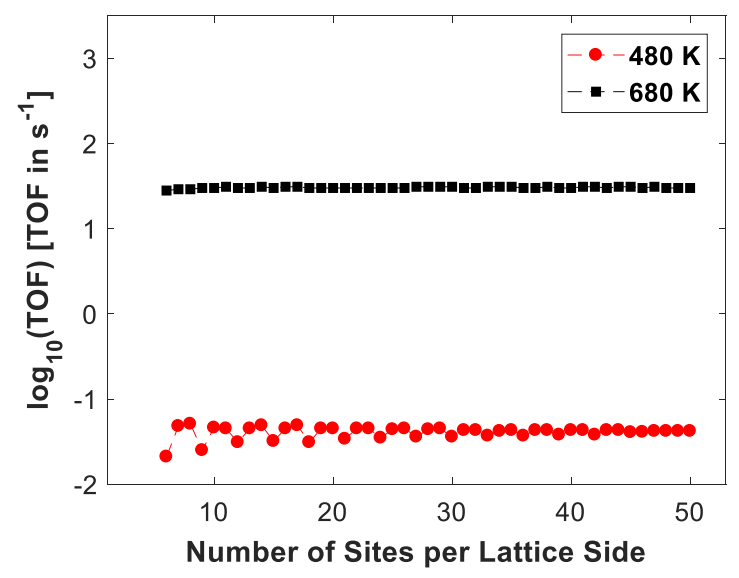

FIG. 7. Calculated TOFs of the 5-Fig. CE model for a range of equilateral parallelogram periodic lattices at $480 \mathrm{~K}$ (red) and $680 \mathrm{~K}$ (black). $\mathrm{P}_{\text {tot }}=1 \mathrm{bar}$, $\mathrm{P}_{\mathrm{NO}_{2}} / \mathrm{P}_{\mathrm{NO}}=0.37$, and $\mathrm{y}_{\mathrm{O}_{2}}=0.1$ for all simulations.
FIG. 6. (a) and (b) show the KMC snapshots of a $44 \times 44$ periodic lattice for 5-Fig. CE energetics at $480 \mathrm{~K}$ and $680 \mathrm{~K}$, respectively. The snapshots are colorcoded according to the $\mathrm{O}_{2}$ dissociation activation energy where blue and red correspond to low and high values, respectively. Panel (c) shows a histogram of the distribution of $\mathrm{O}_{2}$ dissociation activation energies at $480 \mathrm{~K}$ (blue) and $680 \mathrm{~K}$ (green). $\mathrm{P}_{\text {tot }}=1 \mathrm{bar}$, $\mathrm{P}_{\mathrm{NO}_{2}} / \mathrm{P}_{\mathrm{NO}}=0.37$, and $\mathrm{y}_{\mathrm{O}_{2}}=0.1$ bar. is not observed on non-periodic lattices, whereon we compute a virtually constant TOF on larger lattices than $18 \times 18$ (see Fig. S4 of the supplementary material).

To clarify the causes of the lower TOF on smaller commensurate lattices, within the size range of $6 \times 6$ to $45 \times 45$, we investigate the local adlayer configurations for $\mathrm{O}_{2}$ dissociation events. Figures 8(a)-8(d) show the activation energy distributions of the executed $\mathrm{O}_{2}$ dissociations on a $9 \times 9,12$ $\times 12,45 \times 45$, and $48 \times 48$ lattice, respectively. It is important to point out that in this case, the histograms were constructed after processing the statistics of the activation barriers of all occurring $\mathrm{O}_{2}$ dissociations. The main observation is that in all lattices, this elementary event is executed at roughly the same activation energies (i.e., on the same local adlayer configurations). The activation barrier distributions of the two large commensurate lattices [ $45 \times 45$ and $48 \times 48$-Figs. 8 (c) and $8(d)$ ] are identical, and consequently, the two calculated TOFs are in excellent agreement.

Conversely, remarkable differences are noticed in the distributions of the two small lattices (i.e., $9 \times 9,12 \times 12$ ). As seen, on the $9 \times 9$ lattice, around $53 \%$ of $\mathrm{O}_{2}$ adsorptions happen with an activation barrier less than $0.48 \mathrm{eV}$, whilst the corresponding normalized frequency of the next largest commensurate lattice $(12 \times 12)$ is higher by approximately $7 \%$. This difference suggests that local low $\mathrm{O}^{*}$ coverage configurations (i.e., configurations where $\mathrm{O}_{2}$ dissociation can occur with an activation energy of $0.48 \mathrm{eV}$ or less) may be more frequently formed during the KMC simulation as compared to the $9 \times 9$ lattice, thereby explaining the discrepancy between the predicted catalytic rates on the two lattices (Fig. 7).

To verify this speculation, we examine how the catalytic rate is affected by the frequency of configurations where $\mathrm{O}_{2}$ can dissociate with barrier less than $0.48 \mathrm{eV}$ (we refer to these as the "low-barrier" events). This normalized frequency is calculated as follows: we collect KMC lattice snapshots just before 

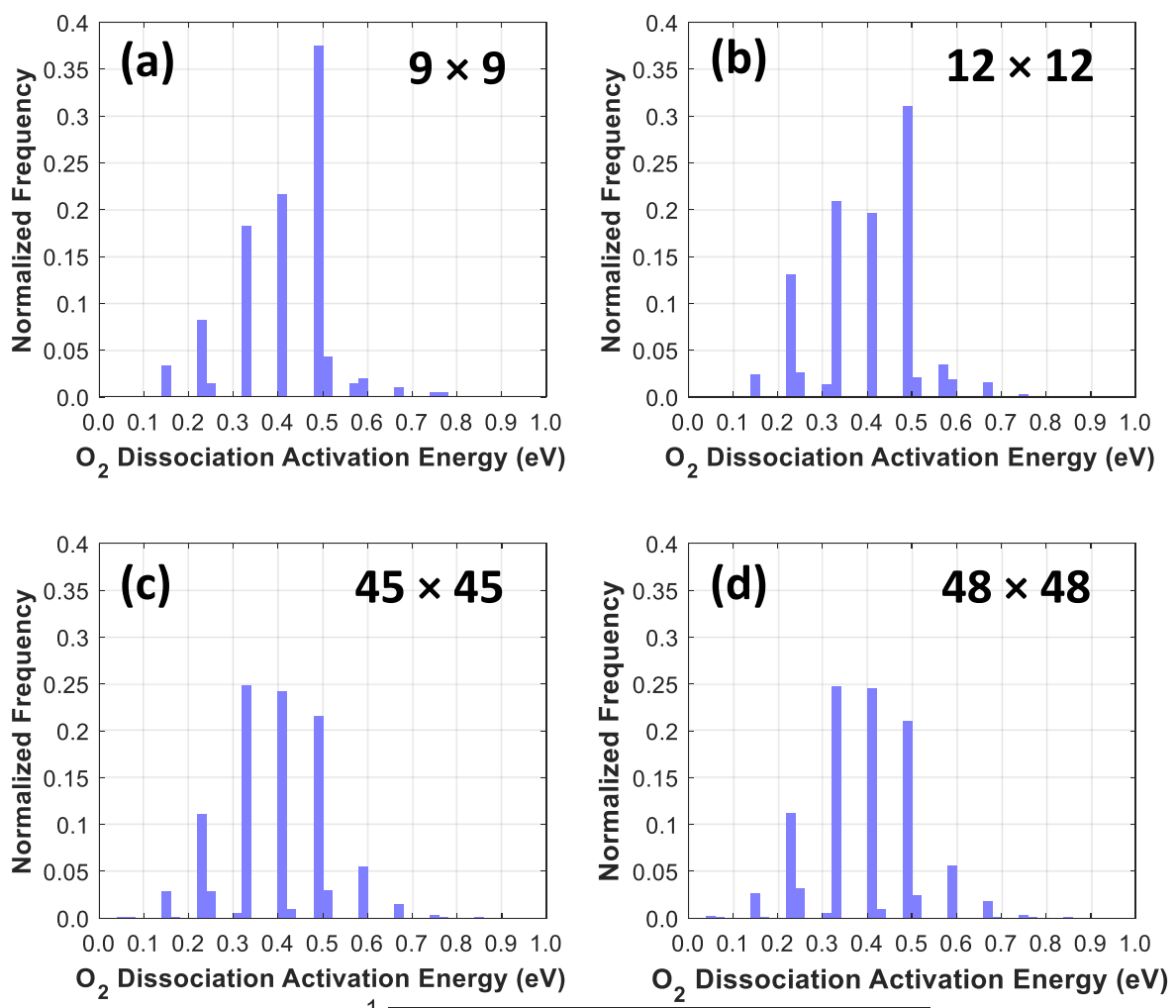

FIG. 8. Panels (a) and (b) show the activation energy distributions of occurring $\mathrm{O}_{2}$ dissociations for 5-Fig. $\mathrm{CE}$ on a $9 \times 9$ and a $12 \times 12$ periodic lattice, respectively. (c) and (d) show the same distribution for a $45 \times 45$ and a $48 \times 48$ periodic lattice, respectively. Panel (e) shows the frequency of appearance of highly active adlayer configurations for nine commensurate lattices. $\mathrm{T}=480 \mathrm{~K}$, $\mathrm{P}_{\text {tot }}=1$ bar, $\mathrm{P}_{\mathrm{NO}_{2}} / \mathrm{P}_{\mathrm{NO}}=0.37$, and $\mathrm{y}_{\mathrm{O}_{2}}=0.1$.

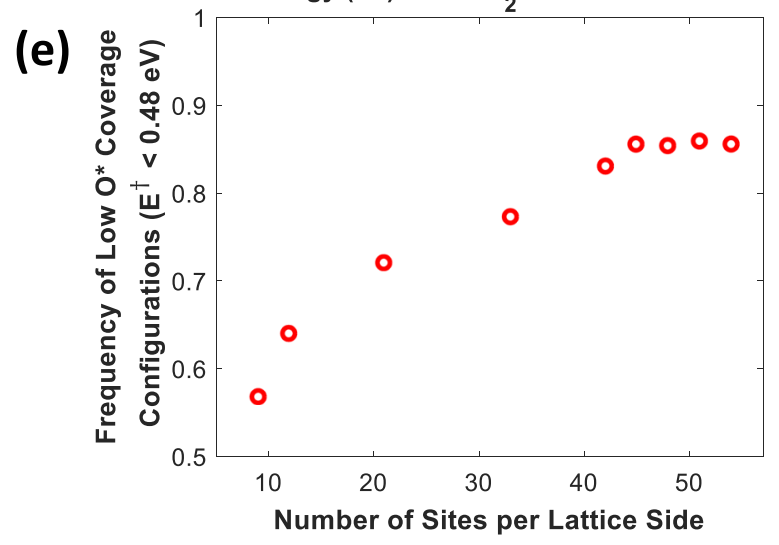

$\mathrm{O}_{2}$ dissociation events. We then count the snapshots that have at least one "low-barrier" $\mathrm{O}_{2}$ dissociation event and divide by the total number of KMC snapshots (i.e., the total number of $\mathrm{O}_{2}$ dissociations). The results for nine commensurate periodic lattices with up to 2916 sites are shown in Fig. 8(e). On lattices smaller than $45 \times 45$, the frequency of highly active configurations increases with the lattice size, whereas for larger lattices there is a convergence of this frequency value to $\sim 0.85$. Interestingly, the observed trend is in excellent agreement with the TOF trend in Fig. 7, where constant catalytic rate values $\left(\mathrm{TOF} \approx 0.042 \mathrm{~s}^{-1}\right)$ are reached on lattices larger than $42 \times 42$.

We finally present the results of an 8-Fig. CE model where $4 \mathrm{NN}$ and $5 \mathrm{NN}$ pairwise interactions as well as a 3-body interaction are taken into account (graph patterns in Fig. 1). At $480 \mathrm{~K}$ and under stationary conditions, a substantial part of the $\mathrm{O}^{*}$ adsorbate layer adopts a $2 \times 2$ structure at surface coverage approximately equal to $0.28 \mathrm{ML}$ [see the $\mathrm{O}_{2}$ dissociation color-coded KMC snapshot in Fig. 9(b)]. Adsorbate-adsorbate interactions become "softer" as compared to 3- and 5-Fig. $\mathrm{CE}$ models. This is reflected in the fact that $\mathrm{O}_{2}$ dissociations can happen on pairs of fcc sites encompassed by the ordered $2 \times 2$ phase with low activation energy being in the range 0.55-1 eV [Fig. 9(b)]. Besides, we observe the formation of several 1-1-3 triplets in the adlayer that, to a limited extent, cause the development of a meandering phase with $2 \times 1 \mathrm{O}^{*}$ domains [Fig. 9(b)]. We find, however, that this structure becomes dominant at higher surface coverage (Fig. S5 in the supplementary material) and therefore these triplets can be considered the precursor of the $2 \times 1 \mathrm{O}^{*}$ phase.

Importantly, because of the largely ordered adlayer at $480 \mathrm{~K}$, the computed TOFs on periodic lattices with size in the range of $10 \times 10$ to $50 \times 50$ [Fig. 9(c)] show again an oscillatory behavior similar to that observed in Fig. 2. Commensurate to the $2 \times 2 \mathrm{O}^{*}$ phase are now lattices with the number of sites (per side) divisible by 2 , and after thorough inspection of event-wise KMC snapshots of commensurate and non-commensurate lattices, we find that there is no formation of well-defined anti-phase boundaries. To this end, the calculated TOFs on different lattices are on the same order of magnitude [Fig. 9(c)], which was not true for the 3-Fig. 

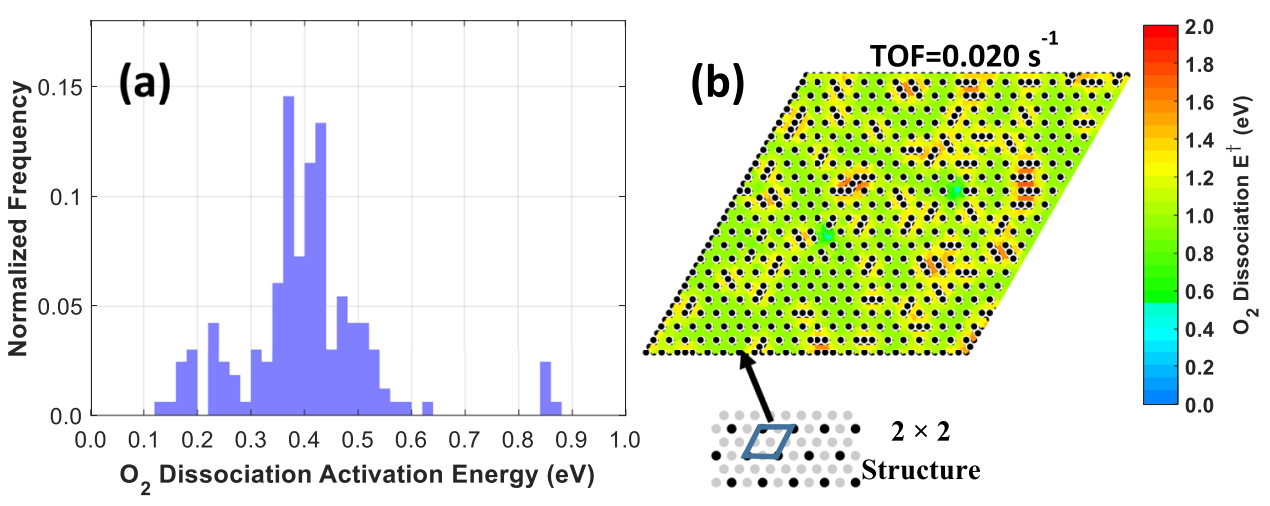

(c)

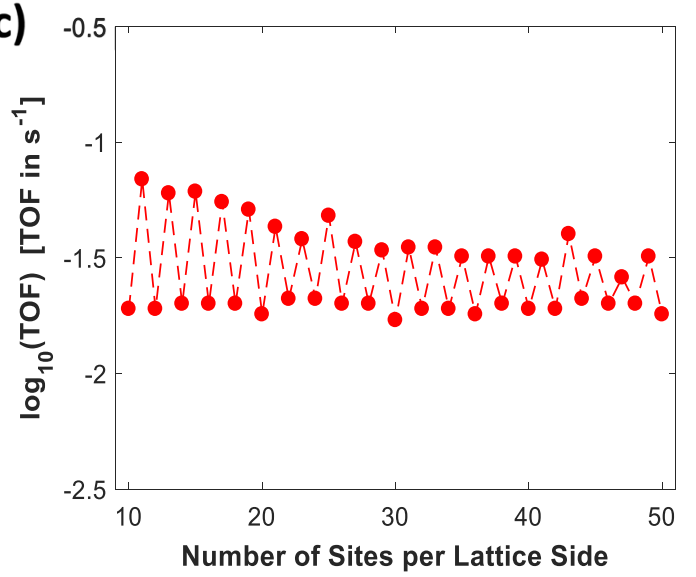

FIG. 9. Panel (a) shows the $\mathrm{O}_{2}$ dissociation activation energy distribution on a $42 \times 42$ commensurate periodic lattice. Panel (b) shows an $\mathrm{O}_{2}$ dissociation KMC color-coded snapshot of the $42 \times 42$ lattice for the 8-Fig. CE energetic model. Panel (c) plots the computed TOF of the 8-Fig. CE model for a range of equilateral parallelogram periodic lattices. $\mathrm{T}=480 \mathrm{~K}, \mathrm{y}_{\mathrm{O}_{2}}=0.1 \mathrm{bar}$, $\mathrm{P}_{\mathrm{tot}}=1$ bar, and $\mathrm{P}_{\mathrm{NO}_{2}} / \mathrm{P}_{\mathrm{NO}}=0.37$.
CE model because of the existence of "hard" short range interactions and therefore anti-phase boundaries.

The two aforementioned adlayer patterns have been detected using scanning tunneling electron microscopy (STEM) and low-energy electron diffraction (LEED) at similar coverages to those reported here ${ }^{40,42}$ and have also been reproduced in computational studies that employ equilibrium models. ${ }^{30,48,49}$ This furnishes evidence that the $\mathrm{O}^{*}-\operatorname{Pt}(111)$ system, at least with regard to the adlayer structure, is more accurately described by an 8-Fig. CE energetic model than by the 3-Fig. CE and 5-Fig. CE models. We thus continue our analysis with a comparison between the apparent activation energy, $E_{\text {app }}^{\ddagger}$, found by the 8-Fig. CE model and experimentally obtained values. ${ }^{50}$

To determine $\mathrm{E}_{\mathrm{app}}^{\ddagger}$, we calculate seven TOFs at different temperatures in the temperature range of $480-493 \mathrm{~K}$ where the differences in surface coverage are known to be small. ${ }^{22}$ From the Arrhenius plot in Fig. 10, we find $E_{\text {app }}^{\ddagger}=52.6$ $\pm 4.0 \mathrm{~kJ} \mathrm{~mol}^{-1}$, which is slightly greater than the reported value by Smeltz et al. ${ }^{50}$ over $\operatorname{Pt}(111)$ single crystals under similar conditions $\left(\sim 41 \mathrm{~kJ} \mathrm{~mol}^{-1}\right)$. Furthermore, we compute TOF $=0.013 \mathrm{~s}^{-1}$ at $\mathrm{P}_{\mathrm{NO}_{2}} / \mathrm{P}_{\mathrm{NO}}=0.56, \mathrm{y}_{\mathrm{O}_{2}}=0.1$ and $480 \mathrm{~K}$. At these conditions, the experimentally reported TOF $\left(\sim 0.15 \mathrm{~s}^{-1}\right)$ is found one order of magnitude larger. ${ }^{50}$ This discrepancy is in accord with the larger computed $\mathrm{E}_{\mathrm{app}}^{\ddagger}$, by $11 \mathrm{~kJ} / \mathrm{mol}(0.1 \mathrm{eV})$, as compared to the experiment. ${ }^{50}$ More specifically, a larger activation barrier by $0.1 \mathrm{eV}$ results in a smaller rate constant by a factor of approximately 11 at $480 \mathrm{~K}$. Errors of around 0.1 to $0.2 \mathrm{eV}$ are indeed expected for commonly used density functional theory (DFT) functionals. ${ }^{51}$
The formation of the $2 \times 2$ phase is explained by the very small energetic contribution parameter of $3 \mathrm{NN}$ clusters $(0.012 \mathrm{eV})$ compared to other clusters in 8-Fig. CE models (see Table $\mathrm{S} 1$ in the supplementary material), leading to the formation of numerous such patterns in the adsorbate layer [Fig. 9(b)]. According to experimental studies, the $2 \times 2 \mathrm{O}^{*}$ phase remains inactive to the catalytic NO oxidation within the temperature range of $350-500 \mathrm{~K}$ at $1 / 4 \mathrm{ML}$ surface coverage, ${ }^{40}$ and this is further corroborated by our 8-Fig. CE simulations. In more precise terms, the activation barrier distribution of the executed $\mathrm{O}_{2}$ dissociations on a $42 \times 42$ periodic lattice

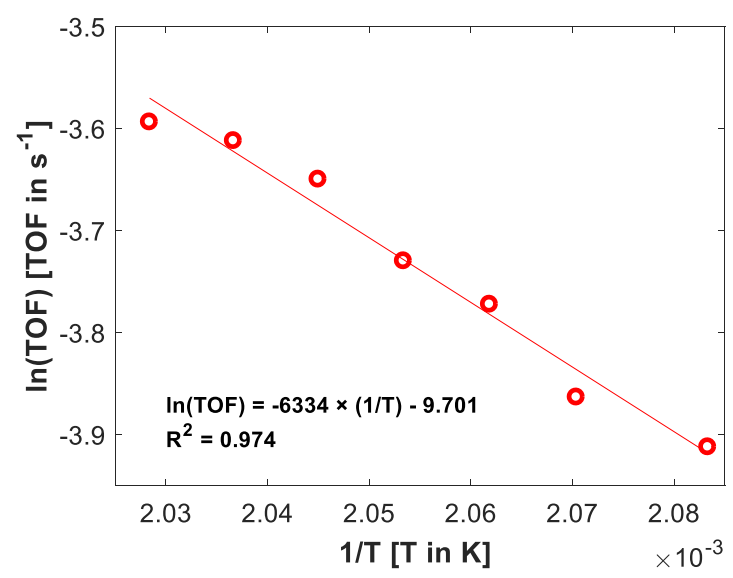

FIG. 10. Arrhenius plot of the temperature dependence of the rate of NO oxidation on a $42 \times 42$ commensurate periodic lattice for 8 -Fig. CE, $\mathrm{y}_{\mathrm{O}_{2}}=0.1$ bar, $\mathrm{P}_{\mathrm{NO}_{2}} / \mathrm{P}_{\mathrm{NO}}=0.37$, and $\mathrm{P}_{\text {tot }}=1$ bar. Slope in $\mathrm{K}, k_{B}$ in $\mathrm{eV} / \mathrm{K}$, and $\mathrm{E}_{\text {app }}^{\ddagger}$ in $\mathrm{eV}$. 
[Fig. 9(a)] reveals that less than $5 \%$ of such events take place with an activation barrier of $0.55-1 \mathrm{eV}$. By contrast, we see that the vast majority of $\mathrm{O}_{2}$ dissociation events happen with barriers below $0.5 \mathrm{eV}$ [Fig. 9(a)]. The contribution of fcc site pairs to the catalytic rate is even more widespread than in the simulations with the 5-Fig. CE. Yet, the overall reaction progresses in a similar manner in both cases with $\mathrm{O}_{2}$ dissociations occurring for various adlayer configurations and not only on highly active point defects as for the 3-Fig. CE simulations. The calculated TOFs are in turn of the same order of magnitude, but not identical, owing to the fact that the adsorbate layer exhibits different structures in the two cases.

\section{CONCLUSIONS}

KMC simulation incorporating BEP relations and CE Hamiltonians for capturing coverage effects is a powerful tool for modeling reactions on solid surfaces, enabling detailed representations of the highly complex adlayer behavior encountered in these systems. In this work, such an approach has been adopted to model the catalytic oxidation of NO on the $\mathrm{Pt}(111)$ facet, investigating CEs of increasing complexity.

We have demonstrated that energetic models including only short-range "hard" interactions result in highly ordered adsorbate overlayer structures. This can also be true for longrange interaction models (e.g., 8-Fig. CE). Under these circumstances, the lattice size is of crucial importance; if the lattice is non-commensurate to the lowest energy adlayer structure, the system will form defective regions and perhaps anti-phase boundaries, which have a significant impact on the reaction kinetics by giving rise to short-lived highly reactive configurations. The latter result in markedly higher TOF values than those of commensurate lattices, giving rise to lattice size dependent catalytic rates. Such effects are related to the lattice periodicity and are not present when using non-periodic lattices, which capture nanoparticle facets.

Moreover, accounting for lateral interaction terms beyond $1 \mathrm{NN}$ can result in higher disorder in the spatial distribution of adsorbates in the adlayer, and generally weaker spatial correlations, although ordered domains are still observed to some extent for 5-Fig. CE and to a large extent for 8-Fig. CE energetics at $480 \mathrm{~K}$. Interestingly, in the latter case, a significant portion of the $\mathrm{O}^{*}$ adlayer adopts a $2 \times 2$ structure, and we again simulate a size dependent catalytic rate for lattices up to $50 \times 50$. Still though, we highlight that in both cases the "soft" interactions lead to an "evenly distributed" occurrence of reaction events in both space and time.

These observations have important implications on two aspects: first, they demonstrate that in systems with an ordered adsorbate overlayer, the strong correlation in the adlayer can lead to interesting effects, whereby the geometry of the domain alone can strongly influence the kinetics. This was clearly demonstrated by the 3-Fig. CE and 8-Fig. CE simulations on periodic lattices, which yield significant differences in TOF between commensurate and non-commensurate lattices. On non-periodic lattices, such a geometric effect was shown to lead to spatial variability in the TOF, even if all sites are chemically equivalent (i.e., adsorbates exhibit the same adsorption energies on all these sites). Second, the accuracy with which the $\mathrm{CE}$ is fitted to density functional theory data ${ }^{32}$ can have a decisive effect on the physics reproduced by KMC simulations, even at the qualitative level. Note that all CEs used in this work had been fitted to the same DFT data; ${ }^{30}$ the only difference was the truncation level, including up to $1 \mathrm{NN}$ interactions in the 3-Fig. CE and up to 3NN interactions in the 5-Fig. CE. For this system, a CE with only $1 \mathrm{NN}$ interactions leads to TOF values that are very different from those of more accurate CEs, and our detailed analysis explains the origin of such deviations.

Finally, our study elucidates that, independent of the lattice type used in KMC simulations (i.e., periodic or nonperiodic), for relatively small lattices, the calculated TOF value will be strongly influenced by the lattice size. More importantly, as periodic lattices are commonly used in such simulations, ${ }^{9,22,29,52}$ one has to be aware of relevant effects that can influence computed catalytic rates, as those discussed herein. We have shown that this can be accomplished through preliminary lattice size testing, as well as careful monitoring of the lattice state and the activation energy distributions during the KMC simulation. As KMC simulations become increasingly popular in the computational catalysis community, these are significant factors that must be taken into consideration for accurate kinetic modeling.

\section{SUPPLEMENTARY MATERIAL}

See supplementary material for details on how the TOF is calculated, how anti-phase boundaries are formed, additional calculations of 5-Fig. CE and 8-Fig. CE models, and a discussion on the commensurability of lattice structures. The Brønsted-Evans-Polanyi and energetic contribution parameters can also be found therein, along with a short discussion on the implementation of BEP relations.

\section{ACKNOWLEDGMENTS}

M.T.D. is supported by the EPSRC Doctoral Prize Fellowship, Grant Reference No. EP/N509577/1. The development of Zacros KMC software has been partially supported by the embedded Computer Science and Engineering (eCSE) program of the ARCHER United Kingdom National Supercomputing Service (Grant Nos. eCSE01-001 and eCSE10-8) and the Leverhulme Trust (Grant No. RPG-2017-361). The authors gratefully acknowledge the use of the UCL High Performance Computing Facility Legion@UCL and associated support services, in the completion of the simulations of this work. We would also like to thank Dr. Miguel Pineda for fruitful discussions and Ms Elmira Hamidova for her work during the early stages of this investigation.

${ }^{1}$ I. Chorkendorff and J. W. Niemantsverdriet, Concepts of Modern Catalysis and Kinetics (Wiley-VCH Verlag, Weinheim, 2003).

${ }_{2}^{2}$ S. Uemiya, N. Sato, H. Ando, T. Matsuda, and E. Kikuchi, Appl. Catal. 67, 223 (1990).

${ }^{3}$ G. Weickert, G. B. Meier, J. T. M. Pater, and K. R. Westerterp, Chem. Eng. Sci. 54, 3291 (1999).

${ }^{4}$ C. G. Yiokari, G. E. Pitselis, D. G. Polydoros, A. D. Katsaounis, and C. G. Vayenas, J. Phys. Chem. A 104, 10600 (2000).

${ }^{5}$ W. H. Weinberg and K. A. Fichthorn, J. Chem. Phys. 95, 1090 (1998). 
${ }^{6}$ M. Stamatakis, J. Phys.: Condens. Matter 27, 13001 (2015).

${ }^{7}$ M. Stamatakis and D. G. Vlachos, ACS Catal. 2, 2648 (2012).

${ }^{8}$ M. Stamatakis, M. A. Christiansen, D. G. Vlachos, and G. Mpourmpakis, Nano Lett. 12, 3621 (2012).

${ }^{9}$ A. P. J. Jansen, An Introduction to Kinetic Monte Carlo Simulations of Surface Reactions (Springer, Heidelberg, 2012).

${ }^{10} \mathrm{~K}$. Binder and D. P. Landau, "Monte Carlo calculations on phase transitions in adsorbed layers," in Advances in Chemical Physics: Molecule Surface Interactions, edited by K. P. Lawley (Wiley, 2007), Vol. 76, pp. 91-152.

${ }^{11}$ D. J. Liu and J. W. Evans, Prog. Surf. Sci. 88, 393 (2013).

${ }^{12}$ W. H. Weinberg, Annu. Rev. Phys. Chem. 34, 217 (1983).

${ }^{13}$ D. J. Liu, A. Garcia, J. Wang, D. M. Ackerman, C. J. Wang, and J. W. Evans, Chem. Rev. 115, 5979 (2015).

${ }^{14}$ C. G. M. Hermse and A. P. J. Jansen, in Catalysis, edited by J. J. Spivey and K. M. Dooley (Royal Society of Chemistry, London, 2006), Vol. 19, pp. 109-163.

${ }^{15}$ M. Nagasaka, H. Kondoh, I. Nakai, and T. Ohta, J. Chem. Phys. 126, 44704 (2007).

${ }^{16}$ D. Y. Murzin, Ind. Eng. Chem. Res. 44, 1688 (2005).

${ }^{17}$ J. Wintterlin, Chaos 12, 108 (2002).

${ }^{18}$ S. Völkening and J. Wintterlin, J. Chem. Phys. 114, 6382 (2001).

${ }^{19}$ J. Méndez, S. H. Kim, J. Cerdá, J. Wintterlin, and G. Ertl, Phys. Rev. B: Condens. Matter Mater. Phys. 71, 085409 (2005).

${ }^{20}$ D. H. Parker, M. E. Bartram, and B. E. Koel, Surf. Sci. 217, 489 (1989).

${ }^{21}$ S. Piccinin and M. Stamatakis, ACS Catal. 4, 2143 (2014).

${ }^{22}$ C. Wu, D. J. Schmidt, C. Wolverton, and W. F. Schneider, J. Catal. 286, 88 (2012).

${ }^{23}$ M. G. Evans and M. Polanyi, Trans. Faraday Soc. 34, 11 (1938).

${ }^{24}$ S. Ovesson, B. I. Lundqvist, W. F. Schneider, and A. Bogicevic, Phys. Rev. B: Condens. Matter Mater. Phys. 71, 115406 (2005).

${ }^{25}$ C. Schaefer and A. P. J. Jansen, J. Chem. Phys. 138, 54102 (2013).

${ }^{26}$ E. Hansen and M. Neurock, Surf. Sci. 441, 410 (1999).

${ }^{27}$ E. S. Hood, B. H. Toby, and W. H. Weinberg, Phys. Rev. Lett. 55, 2437 (1985).

${ }^{28}$ D. Menzel, H. Pfnür, and P. Feulner, Surf. Sci. 126, 374 (1983).

${ }^{29}$ I. Farbman, M. Asscher, and A. Ben-Shaul, J. Chem. Phys. 104, 5674 (1996).
${ }^{30}$ D. J. Schmidt, W. Chen, C. Wolverton, and W. F. Schneider, J. Chem. Theory Comput. 8, 264 (2012).

${ }^{31}$ J. M. Sanchez, F. Ducastelle, and D. Gratias, Physica A 128, 334 (1984).

${ }^{32}$ S. D. Miller and J. R. Kitchin, Mol. Simul. 35, 920 (2009).

${ }^{33}$ M. Pineda and M. Stamatakis, J. Chem. Phys. 147, 24105 (2017).

${ }^{34}$ S. Piccinin and M. Stamatakis, Top. Catal. 60, 141 (2017).

${ }^{35}$ M. Stamatakis and S. Piccinin, ACS Catal. 6, 2105 (2016).

${ }^{36}$ M. Stamatakis and D. G. Vlachos, J. Chem. Phys. 134, 214115 (2011).

${ }^{37}$ J. Nielsen, M. D'Avezac, J. Hetherington, and M. Stamatakis, J. Chem. Phys. 139, 224706 (2013).

${ }^{38}$ M. Stamatakis, "Advanced Lattice-KMC Simulation Made Easy," http://www.zacros.org, 2013.

${ }^{39}$ A. Voter, Radiation Effects in Solids (Springer Science \& Business Media, 2007), pp. 1-23.

${ }^{40}$ K. Mudiyanselage, C. W. Yi, and J. Szanyi, J. Phys. Chem. C 113, 5766 (2009).

${ }^{41}$ R. B. Getman, W. F. Schneider, A. D. Smeltz, W. N. Delgass, and F. H. Ribeiro, Phys. Rev. Lett. 102, 076101 (2009).

${ }^{42}$ S. P. Devarajan, J. A. Hinojosa, and J. F. Weaver, Surf. Sci. 602, 3116 (2008).

${ }^{43}$ J. M. Hawkins, J. F. Weaver, and A. Asthagiri, Phys. Rev. B 79, 125434 (2009).

${ }^{44}$ M. Stamatakis and D. G. Vlachos, Comput. Chem. Eng. 35, 2602 (2011).

${ }^{45}$ G. Peng and M. Mavrikakis, Nano Lett. 15, 629 (2015).

${ }^{46}$ B. Ni and X. Wang, Adv. Sci. 2, 1500085 (2015).

${ }^{47}$ S. S. Mulla, N. Chen, L. Cumaranatunge, G. E. Blau, D. Y. Zemlyanov, W. N. Delgass, W. S. Epling, and F. H. Ribeiro, J. Catal. 241, 389 (2006).

${ }^{48}$ H. Tang, A. Van Der Ven, and B. L. Trout, Phys. Rev. B: Condens. Matter Mater. Phys. 70, 045420 (2004).

${ }^{49}$ R. B. Getman, Y. Xu, and W. F. Schneider, J. Phys. Chem. B 112, 9559 (2008).

${ }^{50}$ A. D. Smeltz, W. N. Delgass, and F. H. Ribeiro, Langmuir 26, 16578 (2010).

${ }^{51}$ K. Lejaeghere, V. Van Speybroeck, G. Van Oost, and S. Cottenier, Crit. Rev. Solid State Mater. Sci. 39, 1 (2014).

${ }^{52}$ M. Stamatakis, Y. Chen, and D. G. Vlachos, J. Phys. Chem. C 115, 24750 (2011).

${ }^{53}$ I. Farbman, M. Asscher, and A. Ben-Shaul, J. Chem. Phys. 104, 5674 (1996). 